\title{
Zielerreichung im Coaching und die Rolle von Kontextfaktoren
}

\author{
Eine Feldstudie mit Führungskräften
}

\author{
Stephan Hentrich • Andreas Müller • Andreas Zimber · Hansjörg Künzli
}

Online publiziert: 24. September 2015

(C) Die Autor(en) 2015. Dieser Artikel ist auf Springerlink.com mit Open Access verfügbar.

\begin{abstract}
Zusammenfassung Der betriebliche Kontext, in dem ein Coachee arbeitet, könnte Einfluss auf den Erfolg eines Coachings ausüben. Der potenzielle Einfluss von Kontextfaktoren auf Ergebniskriterien im Coaching wurde bisher allerdings empirisch nicht untersucht. Es wurde angenommen, dass spezifische Arbeitsbedingungen, wie u. a. das soziale Klima, die Zielerreichung im Coaching erhöhen können. Die Ergebnisse der über drei Messzeitpunkte durchgeführten Verlaufsstudie mit $N=21$ Coachees belegen positive Wirkungen von Coaching in Bezug auf die Zielerreichung. Darüber hinaus kann die Zielerreichung durch spezifische Kontextfaktoren vorhergesagt werden. Implikationen dieser Ergebnisse für die Praxis werden abschließend diskutiert.
\end{abstract}

Schlüsselwörter Coaching · Evaluation · Zielerreichung · Arbeitsbedingungen

S. Hentrich $(\bowtie) \cdot$ A. Müller $\cdot$ A. Zimber

SRH-Hochschule Heidelberg,

Heidelberg, Deutschland

E-Mail: hentrich@uni-bremen.de

A. Müller

E-Mail: mueller.andreas@de.pwc.com

A. Zimber

E-Mail: Andreas.Zimber@fh-heidelberg.de

S. Hentrich

Zentrum für Klinische Psychologie und Rehabilitation (ZKPR), Universität Bremen,

Bremen, Deutschland

H. Künzli

Zürcher Hochschule für Angewandte Wissenschaften (ZHAW), Zürich, Deutschland

E-Mail: hansjoerg.kuenzli@zhaw.ch
Goal attainment and the role of contextual factors on coaching effects

A three-wave follow up study

Abstract The organizational context in which a coachee works may influence on the outcomes of coaching. However, the potential impact of contextual factors on coaching effects has not been investigated systematically so far. It was hypothesized that specific working conditions, i.e. the social climate, support the goal attainment as well as more general coaching outcomes. Results of the three-wave follow-up study with $N=21$ coachees indicate significant effects of the coachings on the goal attainment reported by the coachees. Additionally, specific working conditions predicted the goal attainment substantially. We conclude by discussing the implications for research and practice.

Keywords Coaching - Evaluation - Goal attainment · Working conditions

Coaching hat im betrieblichen Kontext den Ruf, eine ,innovative Maßnahme der Personalentwicklung für Fach- und Führungskräfte“ (Mäthner et al. 2005, S. 55) zu sein. Über Nutzen und Nachhaltigkeit von Coaching existieren allerdings nur wenig empirisch abgesicherte Erkenntnisse. Zwar liefern erste Studien Hinweise zur Wirksamkeit der Methode, doch ist die absolute Anzahl der Arbeiten zum Thema noch immer gering. Eine breite wissenschaftliche Evaluation der Wirksamkeit von Coaching ist jedoch von zentraler Bedeutung sowohl für die Professionalisierung und Qualitätssicherung von Coaching als auch für die ökonomische Rechtfertigung einer kostenintensiven Personalentwicklungsmaßnahme im Unternehmenskontext (Greif 2008; Künzli 2009; Graf et al. 2015; Groß und Stephan 2015). 
An bereits vorliegenden Studien wird außerdem inhaltlich Kritik geübt (Greif 2008; Künzli 2009): Häufige Bedenken liegen in mangelnder Berücksichtigung der Dreiecksbeziehung zwischen Coach, Coachee und Organisation. Durch den Einfluss des Arbeitsumfeldes unterscheidet sich Coaching schließlich deutlich von anderen Beratungsformen (GCC 2008; Möller und Kotte 2011). Kontextfaktoren des Arbeitsumfelds, aus dem der Coachee stammt, üben zudem potenziell Einfluss auf die Zielerreichung eines Coachings aus, werden aber erstaunlicherweise bisher „nirgends thematisiert" (Künzli 2009, S. 10).

Die vorliegende Untersuchung evaluiert die Wirksamkeit von Coaching vor dem Hintergrund ausgewählter Ergebniskriterien aus der Forschungsliteratur (Greif 2008) in einem Längsschnittdesign. Damit leistet die Arbeit einen wichtigen Beitrag zur Erforschung der Wirksamkeit von Coaching. Es wurde eine Zielerreichungsskala verwendet, welche den Fortschritt der individuellen Anliegen des Coachees im Verlauf des Coaching-Prozesses und darüber hinaus abbilden soll. Eine gezielte Anwendung dieser Skala im realen Coachingkontext mit anschließender Überprüfung der Wirksamkeit unterstreicht die praktische Bedeutung der vorliegenden Arbeit. Zusätzlich wurde der Einfluss von Kontextfaktoren auf den Zielerreichungsgrad überprüft, um erste empirische Ergebnisse zur Bedeutsamkeit des Kontextes im Verlauf von Coaching-Prozessen zu gewinnen. An der Untersuchung haben ausschließlich Führungskräfte teilgenommen. Die Ergebnisse werden kritisch diskutiert und die praktische Bedeutsamkeit herausgearbeitet.

\section{Die Bedeutung von Zielen und Kontextfaktoren im Coaching-Prozess}

Coachingprozesse können anhand des „Generischen Prozessmodells für Coaching“"von Orlinsky und Howard (1986) abgebildet werden. Dieses unterscheidet Input-, Prozess-, Ergebnis- und Kontextvariablen (Abb. 1). Es wird davon ausgegangen, dass Inputvariablen (z. B. die Persönlichkeit des Coachs) die Prozesse, und diese wiederum die Ergebnisse, beeinflussen. Kontextvariablen sind Einflussgrößen aus dem betrieblichen oder nicht-betrieblichen Umfeld eines Coachings, die sich positiv oder negativ auf den Ablauf und die Ergebnisse auswirken können, vom Coach und Coachee in der Regel aber nicht oder nur beschränkt gesteuert werden können. Ihre Wirkung kann sich vor (z. B. Geschäftsstrategie), während (z. B. Unterstützung durch Vorgesetzte) oder nach (z. B. Transferklima) dem Coaching bemerkbar machen (Greif 2008).

Ziele sind Dreh- und Angelpunkt bei der Steuerung von Handlungen (Kleinbeck 2006). In ihnen konkretisieren sich die häufig noch vagen Wünsche. Sie richten die Aufmerksamkeit auf die für den Handlungserfolg notwendigen Ressourcen aus, helfen dabei, unnötige Informationen auszublenden und geben Hinweise zum Handlungsfortschritt. Die Tätigkeit des Coachs besteht darin, seinen Coachee bei der Zielfindung, der Zielkonkretisierung und -umsetzung zu unterstützen und zu einem möglichst hohen Zielerreichungsgrad beizutragen (Grant 2006). Im Coaching dienen Reflexionen der Analyse und Erarbeitung von Zielen und Problemlösungen (Greif 2008).

Studien zeigen, dass Coaching den Coachee bei der Zielerreichung unterstützen kann (Brauer 2005, 2006; Greif 2008; Künzli 2009). Künzli (2005) resümiert, dass Coaching unabhängig der Untersuchungsmethode Wirkungen erzielt. Sechs bei Greif (2008) berichtete Studien ohne Kontrollgruppen-Design zu allgemeinen Bewertungskriterien (Zielerreichungsgrad, Zufriedenheit, Verbesserung des Affekts) zeigen einen hohen erlebten Nutzen von Coaching (Grant 2003; Greif 2008; Künzli 2009; Mäthner et al. 2005; Wasylyshyn 2003). In Kontrollgruppen-Studien offenbart

Abb. 1 Generisches Prozessmodell für Coaching (in Anlehnung an Orlinsky und Howard 1986)

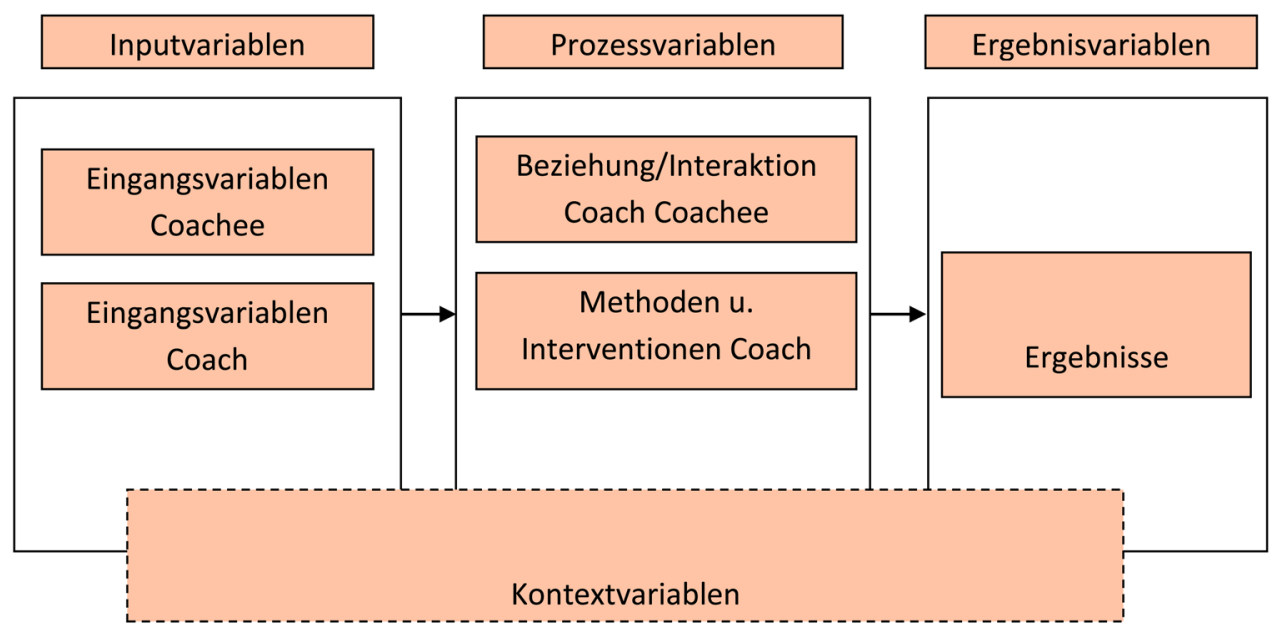


sich ebenfalls eine Erhöhung des Zielerreichungsgrads (Green et al. 2005; Greif 2008; Künzli 2009).

Müller et al. (2004) merken an, dass die Zielerreichung neben internalen Ursachen auch durch äußere Bedingungen (Kontextfaktoren) gehemmt oder unterstützt werden kann. Kontextfaktoren lassen sich in organisationale und soziale Kontextfaktoren differenzieren (Udris 2007; Udris und Rimann 1999). Organisationale Kontextfaktoren umfassen Ressourcen wie den Tätigkeitsspielraum und die Partizipationsmöglichkeiten bei der Arbeit. Der Tätigkeitsspielraum betrifft die Möglichkeiten, Entscheidungen bei der Arbeit zu treffen, sich die Arbeit selbstständig einzuteilen und die gegebenen Ausführungsvarianten bei der Erledigung der Aufgaben. Partizipationsmöglichkeiten spiegeln den Grad wieder, in dem die Firmenleitung, oder der Vorgesetzte rechtzeitig über Änderungen der Arbeitsorganisation informieren und welche Partizipationsmöglichkeiten bei Veränderungen bestehen (Udris und Rimann 1999).

Soziale Kontextfaktoren umfassen Ressourcen wie ein positives Sozialklima, ein mitarbeiterorientiertes Vorgesetztenverhalten und die soziale Unterstützung bei der Arbeit. Positives Sozialklima meint den Grad von gegenseitigem Interesse, Vertrauen, Offenheit und Humor im Umgang mit anderen Personen bei der Arbeit. Mitarbeiterorientiertes Vorgesetztenverhalten charakterisiert das Ausmaß, in dem der Vorgesetzte dem Angestellten bei der Arbeit zugänglich ist, respektvoll und fair gegenübertritt sowie Feedback gibt. Soziale Unterstützung durch den Vorgesetzten beinhaltet das Ausmaß, in dem Vorgesetzte und Kollegen bereit sind, einem Angestellten bzw. einem Arbeitskollegen zuzuhören bei Problemen zu helfen und inwiefern man sich auf sie verlassen kann (Udris und Rimann 1999).

Denkbar ist, dass Kontextfaktoren eine funktionale Zielerreichung im Coaching beeinflussen und die persönliche Weiterentwicklung sowie das Lernen der Coachees stimulieren (Bakker und Demerouti 2006; Bakker et al. 2007; Bakker et al. 2010; Hobfoll 2001).

Es werden folgende Hypothesen aufgestellt:

Hypothese 1: Der Zielerreichungsgrad wird von den Coachees nach dem Coaching höher eingestuft als vor dem Coaching.

Hypothese 2: Je höher Coachees ihren Tätigkeitsspielraum und ihre Partizipationsmöglichkeiten einschätzen, desto höher ist die wahrgenommene Zielerreichung.

Hypothese 3: Je positiver Coachees ihr Sozialklima, das mitarbeiterorientierte Vorgesetztenverhalten und ihre soziale Unterstützung durch Vorgesetzte sowie Kollegen einschätzen, desto höher ist die wahrgenommene Zielerreichung.

\section{Methoden}

\subsection{Stichprobe}

Die Stichprobe setzte sich aus $N=21$ Führungskräften zusammen. Im Verlauf der Studie waren Drop-outs zu verzeichnen (T2: $N=13$; T3: $N=12$ ). Somit lagen Daten von $N=12$ Coachees über alle drei Messzeitpunkte vor. Diese Zielgruppe eignet sich zur Untersuchung des Einflusses von Kontextfaktoren auf den Coachingerfolg, da das Coaching in einem betrieblichen Kontext stattfindet, die Hauptanlässe betrieblicher Art und Führungskräfte die Hauptzielgruppe im Coaching sind (Böning 2006; Böning und Fritschle 2005). Soziodemographische Angaben wurden auf Wunsch der Führungskräfte nicht miterhoben. Die Rekrutierung der Stichprobe erfolgte über Coaching-Ausbildungsinstitute, Verbände und private Kontakte zu Unternehmen. Somit handelt es sich um eine ad hoc-Stichprobe.

\subsection{Untersuchungsdesign und -durchführung}

Die vorliegende Studie entstand im Rahmen eines transnationalen Forschungsprojektes. In einer Feldstudie wurden gecoachte Führungskräfte über drei Messzeitpunkte (Beginn, Ende, Follow-up nach 4-6 Wochen) per Fragebogen befragt. Der Vorteil des Messwiederholungs-Designs liegt in der Kontrolle individueller Störvariablen wie der Veränderungsmotivation (Hertel et al. 2010). Aufgrund fehlender Randomisierung, liegt ein quasi-experimenteller Versuchsplan ohne Kontrollgruppe zugrunde.

Der erste Fragebogen wurde im Anschluss an die erste Coaching-Sitzung, d. h. am Ende der Zielklärungsphase, ausgefüllt (T1). Der zweite Erhebungszeitpunkt fiel in die Endphase des Coachings (T2). Der letzte Messzeitpunkt erfolgte 4-6 Wochen nach dem letzten Coaching-Termin (T3).

\subsection{Messinstrumente}

Die Zielerreichung wurde in Anlehnung an die Goal-Attainment-Scale (GAS) nach Kirusek und Sherman (1968) gemessen. Zunächst wurde nach dem inhaltlichen Ziel gefragt. Die Coachees hatten die Möglichkeit, drei Ziele in der Reihenfolge ihrer persönlichen Bedeutsamkeit anzugeben. Zudem wurde der Zielerreichungsgrad aller drei formulierten Ziele (Zielerreichungsgrad 1, 2 und 3) auf einer elfstufigen Skala in zehner Schritten von 0 bis $100 \%$ erfasst.

Die für das Coaching potenziell relevanten Kontextfaktoren wurden mit dem Fragebogen zur Salutogenetischen Subjektiven Arbeitsanalyse (SALSA) von Udris und Rimann (1999) erfasst. Folgende Skalen aus SALSA wurden herangezogen: Organisationale Ressourcen mit den Subskalen Tätigkeitsspielraum (Beispielitem: „Es ist 
einem genau vorgeschrieben, wie man seine Arbeit machen muss.“), Partizipationsmöglichkeiten und Soziale Ressourcen mit den Subskalen Positives Sozialklima (Beispielitem: „Das gegenseitige Vertrauen ist bei uns so groß, dass wir offen über alles, auch ganz persönliche Sachen, reden können."), Mitarbeiterorientiertes Vorgesetztenverhalten, Soziale Unterstützung durch Vorgesetzte und Kollegen. Die Skalierung verlief von fast nie / trifft überhaupt nicht zu (1) bis fast immer / trifft völlig zu (5) bzw. von gar nicht (1) bis völlig (5).

\subsection{Datenanalyse}

Die Analyse des Einflusses von Kontextfaktoren auf den Zielerreichungsgrad über die Zeit erfolgte mittels Mixedmodels. Als zufällige Effekte gingen die Probanden der Studie in die Modelle ein, damit deren individuelle Ausgangswerte nicht die Analyse beeinflussten. Die Kontextfaktoren, die Messzeitpunkte und die Interaktion zwischen Messzeitpunkt und Kontextfaktoren wurden als feste Effekte modelliert, da systematische Effekte zu erwarten waren. Die Analyse für organisationale und soziale Kontextfaktoren erfolgte voneinander getrennt. Die unabhängigen Variablen wurden sukzessive in das Modell aufgenommen. Die Auswahl der Modelle basiert auf Likelihood-Ratio Tests und der Beurteilung von Informationskriterien (AIC/BIC). Die Prüfung der Normalverteilungsannahme, als Voraussetzung des Verfahrens, erfolgt mittels Shapiro-Francia-Test und Normal-QQ-Plot über die drei Messzeitpunkte hinweg. Zum ersten Messzeitpunkt (T1) lag eine Normalverteilung der Daten zum wichtigsten Zielerreichungsgrad vor; Zielerreichungsgrad 2 und 3 wurden aufgrund einer zu geringen Fallzahl $(N<9)$ ausgeschlossen, da nach Hox (2002) mindestens $N=10$ Fälle pro Beobachtungszeitpunkt vorliegen sollten (Hartig und Rakoczy 2010).

Zur Datenanalyse wurde das Statistikprogramm R (R-Core-Team 2013) mit den Zusatzpaketen lme4 (Bates
Tab. 1 Kategorien zu den angegebenen Coaching-Zielen (inhaltsanalytische Auswertung)

\begin{tabular}{|c|c|c|}
\hline Kategorien & $N$ & Beispielzitate \\
\hline $\begin{array}{l}\text { (1) Persönlich- } \\
\text { keitsentwicklung }\end{array}$ & 8 & Persönliche Stabilisierung \\
\hline $\begin{array}{l}\text { (2) } \\
\text { Karriereplanung }\end{array}$ & 7 & $\begin{array}{l}\text { Unzufriedenheit im Beruf, dadurch } \\
\text { Klärung wo und wie es beruflich wei- } \\
\text { tergehen soll }\end{array}$ \\
\hline $\begin{array}{l}\text { (3) Motivation und } \\
\text { Leistung }\end{array}$ & 7 & $\begin{array}{l}\text { Freier und mit mehr Spaß in die } \\
\text { Akquise gehen }\end{array}$ \\
\hline $\begin{array}{l}\text { (4) Übernahme } \\
\text { neuer Aufgaben } \\
\text { und Funktionen }\end{array}$ & 6 & $\begin{array}{l}\text { Neue Übernahme von } \\
\text { Führungsaufgaben }\end{array}$ \\
\hline $\begin{array}{l}\text { (5) Identi- } \\
\text { fikation mit } \\
\text { Führungsposition }\end{array}$ & 5 & $\begin{array}{l}\text { Bessere Identifikation mit } \\
\text { Führungsposition }\end{array}$ \\
\hline $\begin{array}{l}\text { (6) } \\
\text { Work-Life-Balance }\end{array}$ & 5 & Gute Work-Life-Balance \\
\hline $\begin{array}{l}\text { (7) Konfliktbewäl- } \\
\text { tigung }\end{array}$ & 4 & $\begin{array}{l}\text { Konflikt mit einem Kollegen meiner } \\
\text { Abteilung lösen }\end{array}$ \\
\hline $\begin{array}{l}\text { (8) Teament- } \\
\text { wicklung und } \\
\text { Commitment }\end{array}$ & 2 & $\begin{array}{l}\text { Das Team bei der Teambildung zu } \\
\text { unterstützen }\end{array}$ \\
\hline $\begin{array}{l}\text { (9) Umgang mit } \\
\text { dem Vorgesetzten }\end{array}$ & 2 & $\begin{array}{l}\text { Strategie wie ich mit meiner neuen } \\
\text { Vorgesetzten umgehe (Start mit neuer } \\
\text { Vorgesetzter) }\end{array}$ \\
\hline (10) Sonstiges & 3 & Über Bremsen sprechen \\
\hline
\end{tabular}

$N$ Anzahl Nennungen

et al. 2013) und languageR (Baayen 2011) verwendet. Das Signifikanzniveau wurde auf $5 \%$ festgelegt.

\section{Ergebnisse}

Zunächst werden qualitative und deskriptive Ergebnisse der Gesamtstichprobe vorgestellt: Jeder Coachee hatte die Möglichkeit, drei Ziele zu formulieren und diese nach subjektiver Bedeutsamkeit zu priorisieren. Insgesamt 49 Aussagen zu Coaching-Zielen wurden gewonnen (siehe Tab. 1). Die Coachees gaben in jedem der drei Messzeitpunkte voll-

Tab. 2 Mittelwerte und Standardabweichungen der Untersuchungsvariablen zu den drei Messzeitpunkten

\begin{tabular}{|c|c|c|c|c|c|c|c|c|c|c|c|}
\hline \multirow[b]{2}{*}{ Skala } & \multicolumn{3}{|l|}{$\mathrm{T} 1$} & \multicolumn{4}{|l|}{$\mathrm{T} 2$} & \multicolumn{4}{|l|}{$\mathrm{T} 3$} \\
\hline & $N$ & $M$ & $S$ & $N$ & $M$ & $S$ & $t$ & $N$ & $M$ & $S$ & $t$ \\
\hline (1) Zielerreichungsgrad 1 & 21 & 43,33 & 23,08 & 13 & 65,37 & 25,37 & $6,05^{* * *}$ & 12 & 76,67 & 28,07 & $5,19^{* * *}$ \\
\hline (2) Zielerreichungsgrad 2 & 16 & 55 & 17,89 & 8 & 72,50 & 19,81 & $3,85^{* * *}$ & 8 & 81,25 & 21,66 & $4,92^{* *}$ \\
\hline (3) Tätigkeitsspielraum & 21 & 3,86 & 0,68 & 13 & 4,15 & 0,42 & $4,38^{* * *}$ & 12 & 4,06 & 0,55 & $2,35^{* *}$ \\
\hline (4) Partizipationsmöglichkeiten & 21 & 3,52 & 0,67 & 13 & 3,87 & 0,57 & $2,50^{* *}$ & 12 & 3,85 & 0,74 & 0,65 \\
\hline (5) Positives Sozialklima & 21 & 3,68 & 0,49 & 13 & 3,60 & 0,58 & 0,23 & 12 & 3,68 & 0,76 & 0,69 \\
\hline $\begin{array}{l}\text { (6) Mitarbeiterorientiertes Vorgesetzten } \\
\text { Verhalten }\end{array}$ & 21 & 3,48 & 0,79 & 13 & 3,58 & 0,65 & 1,6 & 12 & 3,62 & 0,76 & $2,65^{* *}$ \\
\hline (7) Soziale Unterstützung Vorgesetzte & 21 & 3,60 & 0,94 & 13 & 3,74 & 0,97 & 0,81 & 12 & 3,67 & 0,88 & $2,02^{*}$ \\
\hline (8) Soziale Unterstützung Kollegen & 21 & 3,94 & 0,79 & 13 & 3,90 & 0,99 & 0 & 12 & 4,03 & 0,98 & 0,23 \\
\hline
\end{tabular}

$\mathrm{t}$-Werte beziehen sich auf den statistischen Vergleich mit T1

${ }^{*} p<.10 ; * * p<.05 ; * * * p<.001$ 


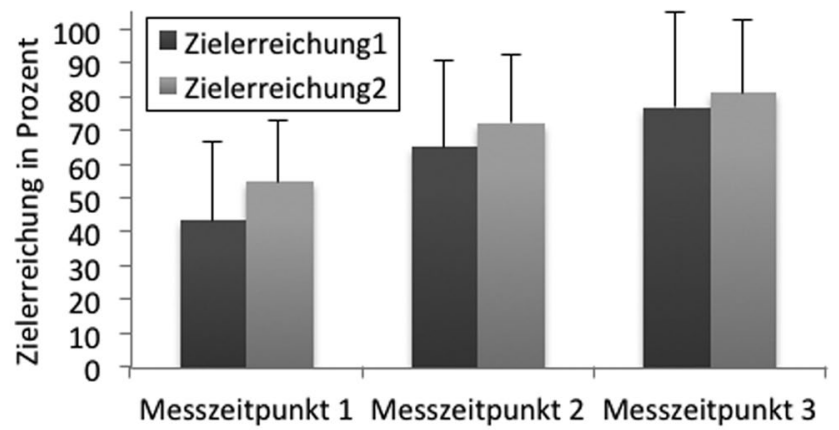

Abb. 2 Veränderungen im Zielerreichungsgrad

ständige Antworten zum wichtigsten Ziel (T1: $N=21 ; \mathrm{T} 2$ : $N=13$; T3: $N=12)$ an. Die Ziele veränderten sich im Verlauf des Coachings inhaltlich nicht.

Zwischen erstem und drittem Messzeitpunkt erhöhen sich die Mittelwerte der Zielerreichungsgrade (siehe Tab. 2 und Abb. 1). Der Zielerreichungsgrad des dritten Ziels wurde aufgrund unzureichender Daten $(N=2)$ von weiteren Analysen ausgeschlossen. Um zu überprüfen, ob sich die Mittelwerte statistisch signifikant unterscheiden, wurden $\mathrm{t}$-Tests berechnet.

Die Zielerreichungsgrade erhöhten sich zwischen T1 und $\mathrm{T} 2$ signifikant (Zielerreichungsgrad $1(t(12)=6.05, p<.001$; siehe Abb. 2) und (Zielerreichungsgrad $2(t(7)=3.85$, $p<.001)$ ). Von T1 zu T3 erhöhten sich die Zielerreichungsgrade ebenfalls signifikant $(t(11)=5.19, p<.001)$ bzw. $(t(7)=4.92, p=.002)$. Auch zwischen T2 und T3 ergaben sich signifikante Veränderungen $(t(10)=2.86, p=.017$ und $(t(7)=2.52, p=.045)$.

An allen Messzeitpunkten wiesen die Skalen eine zufriedenstellende bis sehr gute Reliabilität auf (vgl. Tab. 3 für T3). Alpha variierte in T3 von.68 (Tätigkeitsspielraum) bis.95 (Soziale Unterstützung durch Vorgesetzte). Die Zusammenhänge zwischen Zielerreichung und Kontextfaktoren lagen in erwarteter Richtung. Die Stärken der Koeffizienten sind als mittel bis hoch zu klassifizieren.
Tabelle 4 fasst die Ergebnisse zum Einfluss der Kontextfaktoren auf die Zielerreichung über alle drei Messzeitpunkte zusammen. Beide Modelle unterscheiden sich signifikant vom Nullmodell. Der Haupteffekt für den Tätigkeitsspielraum ist in Modell 1 nach MCMC-Schätzung signifikant. Je höher der Tätigkeitsspielraum ausgeprägt ist, desto höher ist die Zielerreichung, wenn die restlichen Prädiktoren auspartialisiert sind. Dieser Effekt ist um individuelle Ausgangsunterschiede der Coachees bereinigt. Für die Partizipationsmöglichkeiten hingegen ist der Haupteffekt insignifikant. Ebenfalls zeigt sich eine höhere Zielerreichung in T2 und T3 im Vergleich zu T1 (Achsenabschnitt), was auf einen Effekt von Coaching auf die Zielerreichung schließen lässt. Dabei fällt der Effekt in der dritten Erhebungswelle mit 31,76 $(\mathrm{SE}=5,59)\left(\chi^{2}(1)=59,43 \mathrm{p}<.05\right)$ stärker aus als in der Postmessung (T2). Dies spricht für eine nachhaltige Wirkung von Coaching auf die Zielerreichung.

Das Sozialklima leistet in Modell zwei mit 18.87 $(\mathrm{SE}=7.21)$ ebenfalls einen signifikanten Erklärungsbeitrag. Ein günstig eingeschätztes Sozialklima übt, bei Auspartialisierung der restlichen Prädiktoren, einen positiven Einfluss auf die Zielerreichung im Vergleich zu T1 aus. Auch die Haupteffekte der Messzeitpunkte sind signifikant. Die restlichen sozialen Kontextfaktoren (Mitarbeiterorientiertes Vorgesetztenverhalten sowie soziale Unterstützung durch Vorgesetzte und Kollegen) üben nach den vorliegenden Daten keinen bedeutsamen Einfluss auf die Zielerreichung aus.

\section{Diskussion}

Die vorliegende Längsschnitt-Studie evaluiert die Zielerreichung im Führungscoaching mit einer Goal-Attainment-Skala sowie den Einfluss, der hierbei ausgewählten Kontextfaktoren zukommt. Wie die Ergebnisse der Studie zeigen, erhöhte sich zwischen Messzeitpunkt T1 und T2 bei den Coachees die Zielerreichung signifikant, was in Ein-

Tab. 3 Korrelationstabelle zu T3

\begin{tabular}{|c|c|c|c|c|c|c|c|c|c|}
\hline Skala & $N$ & 1 & 2 & 3 & 4 & 5 & 6 & 7 & 8 \\
\hline (1) Zielerreichungsgrad 1 & 12 & - & & & & & & & \\
\hline (2) Zielerreichungsgrad 2 & 8 & 0,58 & - & & & & & & \\
\hline (3) Tätigkeitsspielraum & 12 & $0,62^{*}$ & $0,73^{*}$ & $(0,68)$ & & & & & \\
\hline (4) Partizipationsmöglichkeiten & 12 & $0,74^{*}$ & 0,43 & 0,54 & $(0,74)$ & & & & \\
\hline (5) Positives Sozialklima & 12 & $0,70^{*}$ & 0,59 & 0,58 & $0,62^{*}$ & $(0,80)$ & & & \\
\hline $\begin{array}{l}\text { (6) Mitarbeiter orientiertes Vorgesetzten } \\
\text { Verhalten }\end{array}$ & 12 & 0,39 & 0,49 & $0,75^{*}$ & $0,65^{*}$ & 0,35 & $(0,86)$ & & \\
\hline (7) Soziale Unterstützung durch Vorgesetzte & 12 & 0,42 & 0,26 & 0,59 & $0,78^{* *}$ & 0,37 & $0,94^{*}$ & $(0,95)$ & \\
\hline (8) Soziale Unterstützung durch Kollegen & 12 & 0,28 & 0,56 & 0,27 & 0,50 & 0,35 & 0,24 & 0,13 & $(0,92)$ \\
\hline
\end{tabular}

Reliabilitätskoeffizienten (Cronbachs-Alpha) sind in Klammern berichtet

Korrelationen mit Spearmans-Rho berechnet

${ }^{*} p<05 ; * * p<01$ (zweiseitig) 
Tab. 4 Organisationale und soziale Kontextfaktoren mit Zielerreichung als Kriterium

\begin{tabular}{lll}
\hline Prädiktoren & Modell 1 & Modell 2 \\
\hline Feste Effekte & $B(S E)$ & $B(S E)$ \\
Achsenabschnitt (T1) & $-40,50(27,61)$ & $-25,73(27,03)$ \\
Tätigkeitsspielraum & $19,85^{*}(7,90)$ & - \\
Partizipationsmöglichkeiten & $2,07(6,57)$ & - \\
Soziales Klima & - & $18,78^{* * *}(7,21)$ \\
T2 & $15,78^{*}(5,53)$ & $21,06^{* *}(5,47)$ \\
T3 & $31,76^{* * *}(5,59)$ & $35,67^{* * *}(5,65)$ \\
Zufällige Effekte & & \\
Varianz zwischen den & 316,14 & 313,11 \\
Probanden & & \\
\hline
\end{tabular}

Modelle mit den besten Fit-Indices und signifikanter Unterscheidung zum Nullmodell aufgeführt

$N=21$ Probanden mit $N=43$ Beobachtungen über $N=3$ Messzeitpunkte; p-Werte der $\beta$-Gewichte (nicht standardisierte Werte) sind nach MCMC-geschätzt

${ }^{*} p<.05 ;{ }^{* *} p<.01 ;{ }^{* * *} p<.001$

klang mit anderen Forschungsarbeiten steht (u. a. Böning und Fritschle 2005; Brauer 2005, 2006; Greif 2008; Künzli 2009; Mäthner et al. 2005; Runde und Bastians 2005). Vier Wochen nach dem Coaching ist eine weitere, signifikante Erhöhung der Mittelwerte und eine große Streuung in den Zielerreichungsgraden zu beobachten. Dies spricht dafür, dass Coaching individuell sehr unterschiedliche und nachhaltige Wirkungen entfalten kann (Greif 2008; Künzli 2009; Rauen 2008). Das Ergebnis lässt sich so interpretieren, dass die Coachees während des Coachings gemeinsam mit dem Coach an ihren Zielen arbeiten und dadurch in die Lage versetzt werden, nach dem Coaching erfolgreich weiter an den Zielen zu arbeiten (Hilfe zur Selbsthilfe). Die Ergebnisse lassen in der Gesamtschau darauf schließen, dass Coaching ein nützliches Personalentwicklungsinstrument für Führungskräfte ist, da es wesentlich zur Zielerreichung der Coachees beigetragen hat.

Mixed-models dienten zur Analyse des Einflusses von Coaching und Kontextfaktoren auf den Zielerreichungsgrad. Die Ergebnisse zeigen bedeutsame unmittelbare und persistente Effekte von Coaching auf die Zielerreichung. Wie die Ergebnisse weiterhin zeigen, können Ressourcen einen Einfluss auf Ergebniskriterien im Coaching ausüben. Der Tätigkeitsspielraum und das Sozialklima sind in der Lage, die Zielerreichung vorherzusagen. Dies könnte über die motivationsfördernde und stressreduzierende Funktion von Ressourcen im Coaching-Prozess selbst, oder beim Praxistransfer im Unternehmen erklärt werden (Bakker und Demerouti 2006; Bakker et al. 2007; Bakker et al. 2010; Hobfoll 2001; Hobfoll et al. 2003; Karasek und Theorell 1990; Kauffeld et al. 2008; Xanthopoulou et al. 2007).

Vor dem Hintergrund, dass Künzli (2009) in seinem Review nur zwei Studien mit drei Messzeitpunkten (Finn et al. 2006; Rohmert und Schmidt 2003) präsentiert, liefert die vorliegende Arbeit einen Beitrag zur Forschung bezüglich der Wirksamkeit sowie Nachhaltigkeit von Coaching und der Rolle von Kontextfaktoren.

\subsection{Methodische Einschränkungen und weiterer Forschungsbedarf}

Bisher erheben nur wenige Coaching-Studien Daten zu mehr als zwei Messzeitpunkten (Künzli 2009). Zukünftige Forschung sollte weitere Messzeitpunkte mit längeren und kürzeren Zeitintervallen realisieren, um Erkenntnisse über die dynamische Wirkung von Coaching und Kontextfaktoren zu gewinnen. Dadurch ließen sich Messwiederholungsartefakte kontrollieren (Gollwitzer und Jäger 2009). Da eine Kontrollgruppe fehlt, sind kausale Aussagen über die Wirkung von Coaching nur mit Einschränkungen möglich (Hertel et al. 2010). Die Rekrutierung einer parallelisierten, unspezifisch behandelten Kontrollgruppe im Feld ist schwer zu realisieren. Trotzdem sollte zukünftige Forschung versuchen, längsschnittlich angelegte Feldstudien mit Kontrollgruppen durchzuführen, um noch validere Aussagen über Wirkung und Effekte von Coaching sowie Kontextfaktoren treffen zu können.

Bei der Analyse des Einflusses von organisationalen und sozialen Ressourcen wird deutlich, dass der Tätigkeitsspielraum und das Sozialklima die Zielerreichung bedeutsam vorhersagen. Partizipationsmöglichkeiten, das Vorgesetztenverhalten sowie die soziale Unterstützung scheinen nach den vorliegenden Ergebnissen keinen Einfluss auf die Zielerreichung auszuüben. Untersuchungen mit deutlich größeren Stichproben sind unbedingt notwendig, um die Ergebnisse zu replizieren und zu validieren.

Nur Partnerschaften zwischen Forschung und Praxis können helfen, die Coaching-Forschung weiter auszubauen. Hiervon können Forschung und Praxis profitieren. Gleichwohl ist festzuhalten, dass die vorliegende Arbeit Hinweise mit hoher externer Validität sowohl für die Wirkung von Coaching als auch für die Wirkung von Kontextfaktoren liefert. Abschließend ist zu konstatieren, dass noch erheblicher Forschungsbedarf besteht (Greif 2008; Künzli 2009).

\subsection{Praktische Implikationen}

Coachs sollten Merkmale des Unternehmensumfeldes berücksichtigen. Es wäre vorteilhaft, wenn sich Coachs informieren, wie das gegenseitige Vertrauen am Arbeitsplatz ausgeprägt ist und wie positiv der Coachee seine Handlungsspielräume bei der Arbeit wahrnimmt. Schließlich scheint eine höhere Zielerreichung insbesondere bei einem höheren Tätigkeitsspielraum und einem positiv eingeschätzten Sozialklima erreichbar zu sein. Sind Handlungsfelder identifiziert, sollte der Coach versuchen, mit dem Coachee Lösungen zu finden, um mögliche, aus dem 
(Unternehmens-)Kontext stammende, Zielbarrieren zu reduzieren. Zu Beginn des Coachings könnten Skalen zur Erfassung der Ressourcen des Coachees eingesetzt werden. Hierfür ließe sich ein ökonomischer Kurzfragebogen aus bereits validierten Instrumenten zur Messung entwickeln. Allerdings ist zunächst in weiteren Studien die Akzeptanz solcher Fragebögen sowohl bei Coachees als auch bei Coachs zu untersuchen.

Durch die Verwendung einer Zielerreichungsskala während des gesamten Prozesses ist es nicht nur dem Coachee möglich, seine Zielverfolgung zu kontrollieren. Auch der Coach kann den Fortschritt des Coacheees überprüfen und steuernd eingreifen. Durch die Möglichkeit der Priorisierung der Ziele lassen sich Zielkonflikte vermeiden. Zielkonflikte sind für den Coaching-Verlauf und somit für den Coaching-Erfolg hinderlich (Rauen 2003; Rückle 1992).

Open Access Dieser Artikel wird unter der Creative Commons Namensnennung 4.0 International Lizenz (http://creativecommons. org/licenses/by/4.0/deed.de) veröffentlicht, welche die uneingeschränkte Nutzung, Verbreitung und Wiedergabe für beliebige Zwecke erlaubt, sofern Sie den/die ursprünglichen Autor(en) und die Quelle ordnungsgemäß nennen, einen Link zur Creative Commons Lizenz beifügen und angeben, ob Änderungen vorgenommen wurden.

\section{Literatur}

Baayen, R. H. (2011). Data sets and functions with „Analyzing Linguistic Data: Practical introduction to statistics“. R package (version 1.4.)

Bakker, A. B., \& Demerouti, E. (2006). The job demands-resources model: State of the art. Journal of Managerial Psychology, 22(3), 309-328.

Bakker, A. B., Hakanen, J. J., Demerouti, E., \& Xanthopoulou, D. (2007). Job resources boost work engagement, particularly when job demands are high. Journal of Educational Psychology, 99(2), 274-284.

Bakker, A. B., Van Veldhoven, M., \& Xanthopoulou, D. (2010). Beyond the Demand-Control Model: Thriving on high job demands and resources. Journal of Personnel Psychology, 9(1), 3-16.

Bates, D., Maechler, M., \& Bolker, B. (2013). lme4: Linear mixedeffects models using S4 classes. R package version 0.999999-2. Bezogen über: http://CRAN.Rproject.org/package=lme4. Zugegriffen: 13. Juli. 2013.

Böning, U. (2006). Coaching und supervision: Zur Konkurrenz und Kooperation in der Praxis. In U. Straumann (Hrsg.), Personenzentriertes Coaching und Supervision. Ein interdisziplinärer Balanceakt (S. 228-251). Heidelberg: Asanger.

Böning, U., \& Fritschle, B. (2005). Coaching fürs Business. Bonn: managerSeminare.

Brauer, Y. (2005). Wie Zielvereinbarungen im Coaching helfen. Wirtschaftspsychologie aktuell, 1, 40-43.

Brauer, Y. (2006). Zielvereinbarungen beim Coaching. Eine empirische Untersuchung aus Kundensicht. Düsseldorf: VDM.

Finn, F., Mason, C., \& Griffin, M. (2006). Investigating change over time - The effects of executive coaching on leaders' psychological states and behavior, Paper held at the 26th International Congress of Applied Psychology. Athens, Greece.
Global Coaching Community (GCC). (2008). A research agenda for the development of the field - White paper global convention on coaching. Dublin, Ireland.

Gollwitzer, M., \& Jäger, R. R. (2009). Evaluation Kompakt. Weinheim: Beltz.

Graf, E.-M., Berninger-Schäfer, E., \& Künzli, H. (2015). Zum Start der Zeitschrift: Was ist und welches Ziel verfolgt die Zeitschrift Coaching | Theorie und Praxis? Coaching / Theorie und Praxis.

Grant, A. M. (2003). The impact of life coaching on goal attainment, metacognition and mental health. Social Behavior and Personality, 31(3), 253-264.

Grant, A. M. (2006). An integrative goal-focused approach to executive coaching. In D. R. Stober \& A. M. Grant(Eds.), Evidence based coaching handbook: Putting best practice to work for your clients (S. 153-192). New York: Wiley.

Green, S., Oades, L. G., \& Grant, A. M. (2005). An evaluation of a lifecoaching group program: Initial findings from a waitlist control study. In M. Cavanagh, A. M. Grant \& T. Kem (Hrsg.), Evidence based coaching vol.1: Theory, research and practice from the behavioural sciences (S. 127-141). Bowen Hills Qld: Australian Academic Press.

Greif, S. (2008). Coaching und ergebnisorientierte Selbstreflexion. Göttingen: Hogrefe.

Groß, P.-P., \& Stephan, M. (2015). Der Coaching-Markt. Coaching $\mid T$ heorie und Praxis. doi:10.1365/s40896-015-0002-9.

Hartig, J., \& Rakoczy, K. (2010). Mehrebenenanalyse. In H. Holling \& B. Schmitz (Hrsg.), Handbuch Statistik, Methoden und Evaluation. Göttingen: Hogrefe.

Hertel, S., Klug, J., \& Schmitz, B. (2010). Quasi experimentelle Versuchspläne. In H. Holling \& B. Schmitz (Hrsg.), Handbuch der Psychologie: Statistik, Methoden und Evaluation (S. 49-61). Göttingen: Hogrefe.

Hobfoll, S. E. (2001). The influence of culture, community, and the nested-self in the stress process: Advancing conservation of resources theory. Applied Psychology: An International Review, 50, $337-370$.

Hobfoll, S. E., Johnson, R. J., Ennis, N., \& Jackson, A. P. (2003). Resource loss, resource gain, and emotional outcomes among inner city women. Journal of Personality and Social Psychology, 84(3), 632-643.

Hox, J. J. (2002). Multilevel analysis: Techniques and applications (Bd. Erlbaum). Mawah: Mawah.

Karasek, R. A., \& Theorell, T. (1990). Healthy Work, stress, productivity, and the reconstruction of working life. New York: Basic Books.

Kauffeld, S., Bates, R., Holton, E. F. II, \& Müller, A. C. (2008). Das Lerntransfer-System-Inventar (GLTSI): Psychometrische Überprüfung der deutschsprachigen Version. Zeitschrift für Personalpsychologie, 7(2), 50-69.

Kirusek, T. J., \& Sherman, R. R. (1968). Goal attainment scaling: A general method for evaluating comprehensive community mental health programm. Community Mental Health Journal, 4(6), 443-453.

Kleinbeck, (2006). Handlungsziele. In: J. Heckhausen \& H. Heckhausen (Hrsg.), Motivation und Handeln, 255-276.

Künzli, H. (2005). Wirksamkeitsforschung im Führungskräfte-Coaching. Organisationsberatung - Supervision- Coaching, 12(3), 231-343.

Künzli, H. (2009). Wirksamkeitsforschung im Führungskräfte-Coaching. Organisationsberatung - Supervision- Coaching, 16(1), $1-19$.

Mäthner, E., Jansen, A., \& Bachmann, T. (2005). Wirksamkeit und Wirkung von Coaching. In C. Rauen (Hrsg.), Handbuch Coaching (Bd. 3, S. 55-76). Göttingen: Hogrefe.

Möller, H., \& Kotte, S. (2011). Die Zukunft der Coachingforschung. Organisationsberatung - Supervision- Coaching, 18, 445-456. 
Müller, A., Mohr, G., \& Rigotti, T. (2004). Differenzielle Aspekte psychischer Beanspruchung aus Sicht der Zielorientierung. Die Faktorstruktur der Irritationsskala. Zeitschrift für Differentielle und Diagnostische Psychologie, 25(4), 213-225.

Orlinsky, D. E., \& Howard, K. I. (1986). Process and outcome in psychotherapy. In S. L. Garfield \& A. E. Bergin (Hrsg.), Handbook of psychotherapy and behavior chance (S. 311-384). New York: Wiley.

Rauen, C. (2003). Coaching, Innovative Konzepte im Vergleich. Göttingen: Hogrefe.

Rauen, C. (2008). Coaching. In H. Schuler, R. Hossiep, M. Kleinmann \& W. Sarges (Hrsg.), Praxis der Personalpsychologie (S. 56-66). Göttingen: Hogrefe.

R-Core-Team. (2013). R: A language and environment for statistical computing. R Foundation for Statistical Computing. Austria: Bezogen über: http://www.R-project.org. Zugegriffen: 08. Aug. 2013.

Rohmert, E., \& Schmidt, E. W. (2003). Coaching ist messbar. New management, 1-2, 46-53.
Rückle, H. (1992). Coaching. Düsseldorf: Econ.

Runde, B. \& Bastians, F. (2005). Internes Coaching bei der Polizei NRW - eine multimethodale Evaluationsstudie. Beitrag zum Coaching-Kongress 4.-5.3.2005, Frankfurt/M.

Udris, I. (2007). Gesundheits- und verhaltensbezogene Intervention. In H. Schuler \& K. Sonntag (Hrsg.), Handbuch der Arbeits- und Organisationspsychologie (S. 669-679). Göttingen: Hogrefe.

Udris, I., \& Rimann, M. (1999). SAA und SALSA: Zwei Fragebogen zur subjektiven Arbeitsanalyse. In H. Dunckel (Hrsg.), Handbuch psychologischer Arbeitsanalyseverfahren (S. 397-419). Zürich: vdf Hochschulverlag.

Wasylyshyn, K. M. (2003). Executive coaching: An outcome study. Consulting Psychology Journal: Practice and Research, 55(2), 94-106.

Xanthopoulou, D., Bakker, A. B., Demerouti, E., \& Schaufeli, W. B. (2007). The role of personal resources in the job demands-resources model. International Journal of Stress Management, 14(2), $121-141$. 